This item was submitted to Loughborough's Research Repository by the author.

Items in Figshare are protected by copyright, with all rights reserved, unless otherwise indicated.

\title{
A nonhydrostatic unstructured-mesh soundproof model for simulation of internal gravity waves
}

PLEASE CITE THE PUBLISHED VERSION

http://dx.doi.org/10.2478/s11600-011-0043-z

\section{PUBLISHER}

Versita / ( I Institute of Geophysics, Polish Academy of Sciences

VERSION

AM (Accepted Manuscript)

LICENCE

CC BY-NC-ND 4.0

\section{REPOSITORY RECORD}

Smolarkiewicz, Piotr K., and Joanna Szmelter. 2019. "A Nonhydrostatic Unstructured-mesh Soundproof Model for Simulation of Internal Gravity Waves". figshare. https://hdl.handle.net/2134/13369. 
This item was submitted to Loughborough's Institutional Repository (https://dspace.lboro.ac.uk/) by the author and is made available under the following Creative Commons Licence conditions.

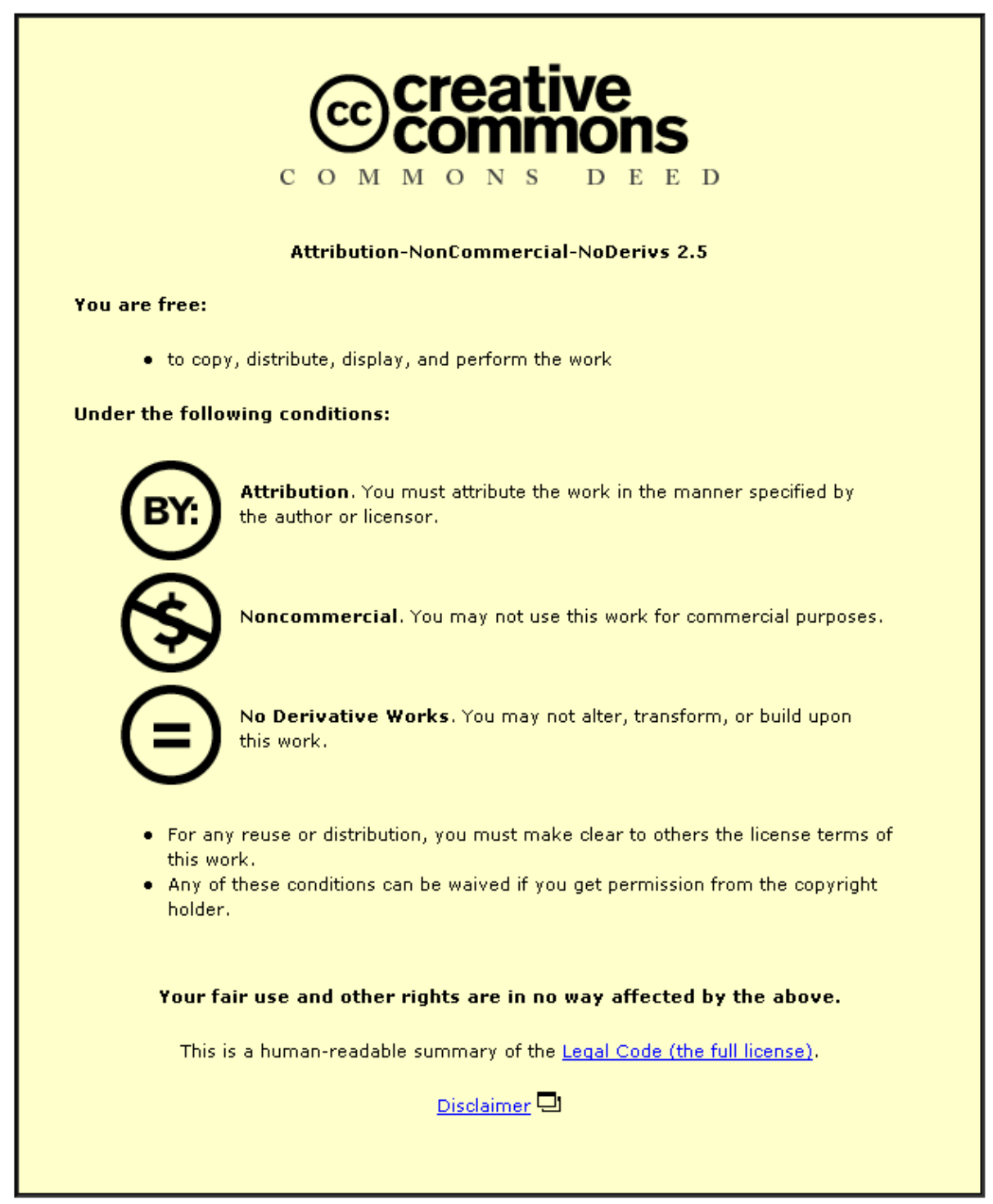

For the full text of this licence, please go to: http://creativecommons.org/licenses/by-nc-nd/2.5/ 


\title{
A Nonhydrostatic Unstructured-Mesh Soundproof Model for Simulation of Internal Gravity Waves
}

\author{
Piotr K. SMOLARKIEWICZ ${ }^{1}$ and Joanna SZMELTER ${ }^{2}$ \\ ${ }^{1}$ National Center for Atmospheric Research \\ Boulder, CO 80307, USA \\ email: smolar@ucar.edu (corresponding author) \\ ${ }^{2}$ Loughborough University \\ Leicestershire LE11 3TU, UK \\ email: j.szmelter@lboro.ac.uk
}

\begin{abstract}
A semi-implicit edge-based unstructured-mesh model is developed that integrates nonhydrostatic soundproof equations, inclusive of anelastic and pseudo-incompressible systems of partial differential equations. The model builds on nonoscillatory forwardin-time MPDATA approach using finite-volume discretization and unstructured meshes with arbitrarily shaped cells. Implicit treatment of gravity waves benefits both accuracy and stability of the model. The unstructured-mesh solutions are compared to equivalent structured-grid results in simulations of an intricate multiscale internal wave phenomenon of a non-Boussinesq amplification and breaking of deep stratospheric gravity waves. The departures of the anelastic and pseudo-incompressible results are quantified in reference to a recent asymptotic theory [Achatz et al., 2010, J. Fluid Mech., 663, 120-147].
\end{abstract}

Key words: unstructured mesh models, nonoscillatory forward-in-time schemes, atmospheric models, soundproof equations, mountain waves 


\section{INTRODUCTION}

All atmospheric models already differ in some aspects at the theoretical level, as to date there is no single, uniformly adopted set of equations governing weather and climate - cf. the collection of works in the special issue Miller and Smolarkiewicz (2008). Compressible dynamics are universally valid across the entire range of spatial and temporal scales, from small-scale turbulence to planetary circulations, yet they impose computational limitations that are difficult to overcome (Klein, 2011). Notwithstanding the dominant opinion that only fully compressible equations are appropriate for predicting weather and climate at nonhydrostatic resolutions, the debate on the preferred formulation of the governing partial differential equations (PDEs) continues. This is evidenced by numerous recent developments consequential for the advancement of nonhydrostatic soundproof models for simulation of weather and climate; cf. Smolarkiewicz (2011) for a review.

Concomitantly, the last decade saw increased interest and numerous developments in modeling atmospheric flows on alternative meshes to regular Cartesian grids common in meteorological models; see Behrens et al. (2010) for a substantiation. Although studies exploring unstructured meshing date back to the nineteen sixties (Williamson, 2008), the interests in flexible mesh adaptivity have emerged more recently - cf. the collection of papers in Nikiforakis (2009) — with the advent of multiscale Earth-system modeling and climate prediction. A notable advancement is the OMEGA model of Bacon et al. (2000) for forecasting high-impact weather, air quality, and environmental hazard; see Bacon et al. (2008) for a recent overview. In spite of a high level of activity, generally, adaptivemesh atmospheric models have not yet met the demands of modern operational weather prediction and climate studies, as reviewed in Miller and Smolarkiewicz (2008). To date, the research into unstructured mesh atmospheric models is largely confined to idealized applications addressing either synoptic flows in the lowest order long-wave approximation governed by the shallow water equations, or small-scale buoyant phenomena in a neutrally stratified quiescent atmosphere simulated with derivatives of Euler equations. Apart from these two diverse classes of motion, there is an abundance of natural multiscale phenomena relevant to weather and climate but rarely addressed with contemporary unstructured-mesh models. In particular, unstructured-mesh simulations of internal gravity waves are scarce. In the Earth's atmosphere these waves are both ubiquitous and intricate, as their occurrence and form depend on a relative magnitude and structure of ambient flow, density/entropy stratification and forcing (Smith, 1979; Wurtele et al., 1996; Doyle et al., 2000). Consequently, their numerical simulation constitutes a canonical aspect of weather-prediction and climate models.

This paper contributes to both aforementioned areas of interest, and it adds to our recent progress with unstructured-mesh modeling of atmospheric waves throughout a range of scales and physical scenarios (Szmelter and Smolarkiewicz, 2010, 2011). In particular, it presents a new computational model that integrates nonhydrostatic soundproof 
equations, inclusive of incompressible Boussinesq, anelastic and pseudo-incompressible systems of PDEs.

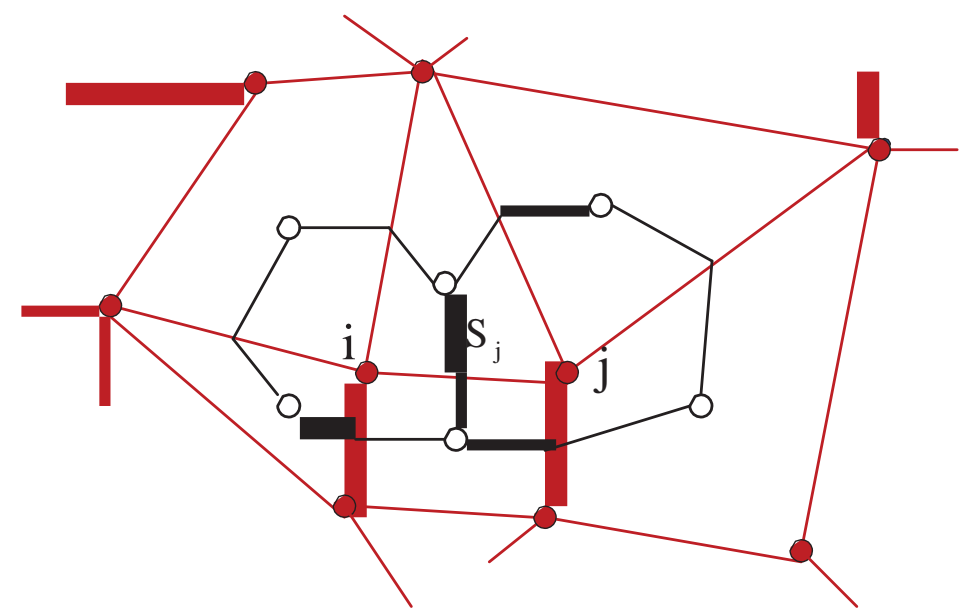

Figure 1: The edge-based, median-dual discretization approach in 2D. The edge connecting vertices (viz. data points) $i$ and $j$ pierces the face $S_{j}$ shared by $2 \mathrm{D}$ computational (dual) cells surrounding vertices $i$ and $j$. Open circles represent centers of the polygonal mesh cells; see (Szmelter and Smolarkiewicz, 2010) for a discussion.

The algorithmic framework used in our unstructured/hybrid mesh models generalizes the methodologies proven in the structured grid model EULAG; see Prusa et al. (2008) for a review. A distinct key element of the framework is the MPDATA suite of nonoscillatory advection schemes - cf. Smolarkiewicz (2006) for a recent overview and history of developments - derived from the first principles for an arbitrary unstructured mesh (Smolarkiewicz and Szmelter, 2005a,b) and implemented for the median-dual finite volume edge-based (Barth, 1992) discretization approach, Fig. $1{ }^{1}$ Remaining key elements of the framework - a robust nonsymmetric Krylov-subspace elliptic solver (Smolarkiewicz et al., 2004) and a class of nonoscillatory forward-in-time algorithms for integrating governing PDEs (Smolarkiewicz and Margolin, 1993; Smolarkiewicz and Szmelter, 2009) closely follow their structured grid predecessors. In EULAG the structured grid nonoscillatory forward-in-time framework is formulated in general ized time-dependent curvilinear coordinates, enabling dynamic grid adaptivity via continuous mappings, in either Cartesian or spherical domains (Prusa and Smolarkiewicz, 2003; Prusa et al., 2008). The same

\footnotetext{
${ }^{1}$ Although the structured-grid MPDATA dates back to the early nineteen eighties (Smolarkiewicz, 1983, 1984), its potential for unstructured-mesh approximations was recognized much later: first in context of mantle convection models (Bunge et al., 1997); and then in the area of weather and environmental modeling (Bacon et al., 2000).
} 
formulation is possible for unstructured meshes - that is, the formulation with the governing equations cast in curvilinear coordinates but discretized on unstructured mesh in a transformed space. The latter is particularly useful for modeling global circulations in a classical geospherical reference frame with the governing equations cast in the latitudelongitude surface-based coordinates (Szmelter and Smolarkiewicz, 2010). Exploiting the flexibility of unstructured meshes allows then to circumvent the notorious limitations associated with the convergence of meridians in the polar regions. Here, we are concerned with local-area modeling of stratified mesoscale flows and use flexible meshing directly in the physical space. This allows us to dispense with terrain-following coordinate transformations (Wedi and Smolarkiewicz, 2004), and better expose the strengths of unstructured meshes.

The following section presents the governing equations underlying the local area nonhydrostatic soundproof model, whereas numerical approximations are discussed in section 3. Section 4 shows a suite of canonical gravity-wave solutions, addressing various aspects (analytic and computational) of the model formulation. Remarks in section 5 conclude the paper.

\section{GOVERNING SOUNDPROOF EQUATIONS}

The focus of this work is on simulation of idealized gravity wave dynamics in absence of viscosity, heat sources or sinks, and planetary rotation. The two alternative governing equations sets manipulated throughout the paper are the anelastic system (Lipps and Hemler, 1982; Lipps, 1990)

$$
\nabla \cdot(\bar{\rho} \mathbf{v})=0, \quad \frac{\mathrm{D} \theta}{\mathrm{Dt}}=0, \quad \frac{\mathrm{D} \mathbf{v}}{\mathrm{Dt}}=-\nabla \Phi^{\prime}-\mathbf{g} \frac{\theta^{\prime}}{\theta},
$$

and the pseudo-incompressible system (Durran, 1989, 2008)

$$
\nabla \cdot(\bar{\rho} \bar{\theta} \mathbf{v})=0, \quad \frac{\mathrm{D} \theta}{\mathrm{Dt}}=0, \quad \frac{\mathrm{D} \mathbf{v}}{\mathrm{Dt}}=-\mathrm{c}_{\mathrm{p}} \theta \nabla \pi^{\prime}-\mathbf{g} \frac{\theta^{\prime}}{\boldsymbol{\theta}} .
$$

The respective definitions of variables and symbols underlying (1) and (2) are as follows: $\rho$ and $p$ denote the density and pressure; whereas $\Phi=p / \bar{\rho}$. Furthermore, $\theta=T\left(p / p_{0}\right)^{-R / c_{p}}$ and $\pi=\left(p / p_{0}\right)^{R / c_{p}}$ are, correspondingly, the potential temperature and the Exner function pressure, with $R, c_{p}$ and $p_{0}$ indicating the gas constant for dry air, specific heat at constant pressure and a constant reference pressure. Vectors $\mathbf{v}=(u, v, w)$ and $\mathbf{g}=(0,0,-g)$ denote the flow velocity and the gravitational acceleration. Primes refer to deviations from a hydrostatic reference state $c_{p} \theta \nabla \bar{\pi} \equiv \bar{\rho}^{-1} \nabla \bar{p}=\mathbf{g}$, so $\theta^{\prime}=\theta-\bar{\theta}, \Phi^{\prime}=(p-\bar{p}) / \bar{\rho}$ and $\pi^{\prime}=\pi-\bar{\pi}$.

There are two noteworthy differences between (1) and (2). First, for a stably stratified atmosphere the effective density profile in the pseudo-incompressible mass continuity 
equation, say $\rho_{\text {eq.2 }}=\bar{\rho}\left(\bar{\theta} / \theta_{0}\right)$ tends to decay slower with altitude than in the anelastic system; here $\theta_{0}$ is a constant reference value. Second, the momentum equation in (2) is unapproximated; whereupon the factor $\theta$ appears in front of the pressure-gradient, thus admitting full, 3D baroclinic production of vorticity. This contrasts with the anelastic momentum equation in (1) that implies the baroclinic vorticity production abbreviated to the horizontal gradient of buoyancy, thus precluding thermally-driven circulations in planes off the vertical. The differences between the pseudo-incompressible and anelastic solutions are small for typical mesoscale tropospheric circulations, but are expected to amplify with increasing stratification and/or with increasing vertical and horizontal scales of addressed atmospheric problems (Davies et al., 2003; Smolarkiewicz and Dörnbrack, 2008; Klein et al., 2010; Achatz et al., 2010). Furthermore, in a small-scale limit, setting $\bar{\rho}=\rho_{0}$ and $\bar{\theta}=\theta_{0}$ reduces (1) and (2) to the incompressible Boussinesq and Euler equations, respectively.

To facilitate the presentation of approximate-solution procedures, we manipulate generic forms (1) and (2) into an "object-oriented" unified system employed in the numerical model. First we note that any inertial ambient state $\mathbf{v}_{\mathrm{e}}(\mathbf{x})$ with $\mathrm{D} \mathbf{v}_{\mathrm{e}} / \mathrm{Dt}=0$ implies compatibility (balance) conditions

$$
0=-\nabla \frac{p_{e}-\bar{p}}{\bar{\rho}}-\mathbf{g} \frac{\theta_{e}-\bar{\theta}}{\theta}
$$

and

$$
0=-c_{p} \theta_{e} \nabla\left(\pi_{e}-\bar{\pi}\right)-\mathbf{g} \frac{\theta_{e}-\bar{\theta}}{\theta},
$$

for (1) and (2), respectively. Second, we subtract (3) and (4) from (1) and (2), correspondingly, to form their unified perturbational equivalent

$$
\nabla \cdot\left(\rho^{*} \mathbf{v}\right)=0, \quad \frac{\mathrm{D} \theta^{\prime}}{\mathrm{Dt}}=-\mathbf{v} \cdot \nabla \theta_{\mathrm{e}}, \quad \frac{\mathrm{D} \mathbf{v}}{\mathrm{Dt}}=-\Theta \nabla \varphi^{\prime}-\mathbf{g Y} \frac{\theta^{\prime}}{\theta}
$$

In (5), respectively for the anelastic and the pseudo-incompressible systems [(1), (2)]: $\rho^{*}=\left[\bar{\rho}, \bar{\rho} \bar{\theta} / \theta_{0}\right] ; \Theta=\left[1, \theta / \theta_{0}\right]$; and $Y=\left[1, \bar{\theta} / \theta_{e}\right]$; while the primes refer now to deviations with respect to the corresponding ambient state, so $\theta^{\prime}=\theta-\theta_{\mathrm{e}}$ and $\varphi^{\prime}=\varphi-\varphi_{\mathrm{e}}$. As far as the solutions are sought for $\mathbf{v}$ and $\theta$, the actual definition of $\varphi$ is flexible, because the pressure variable in soundproof models adapts to the coefficient in front of the gradient via the elliptic boundary problem and the velocity boundary conditions; section 3.2. For diagnostic purposes, however, $\varphi^{\prime}=\left[\left(p-p_{e}\right) / \bar{\rho}, c_{p}\left(\pi-\pi_{e}\right) \theta_{0}\right]$, respectively for [(1), (2)].

There is substantial freedom in specifying ambient states and forming perturbation forms of the governing equations. In essence, the goal is to precondition the governing equations with a particular solution such as to facilitate numerical procedures. In a trivial limit, $\left(\mathbf{v}_{\mathrm{e}}, \theta_{\mathrm{e}}, \varphi_{\mathrm{e}}\right)=(\mathbf{0}, \bar{\theta}, \bar{\varphi})$ and (5) recovers the original systems (1) and (2). Yet in 
general, resorting to perturbation forms of the governing equations can simplify the design of the initial and boundary conditions as well as enhance the accuracy of calculations in finite-precision arithmetics. The ambient states in (3) and (4) are elementary, but once accounted for in the model code, they can be extended to geostropically and thermally balanced global flows (Smolarkiewicz et al., 2001; Grabowski and Smolarkiewicz, 2002), tidal motions (Warn-Varnas et al., 2007), and polytropic stable/unstable background states in solar magneto-convection (Ghizaru et al., 2010).

\section{NUMERICAL APPROXIMATIONS}

\subsection{Prognostic model algorithm}

The two evolutionary equations in (5) are of the form $D \psi / D t=R$, with $\psi$ symbolizing either the potential temperature perturbation or a velocity component and $\mathrm{R}$ depoting the associated right-hand-side (rhs). Accordingly, by combining $\rho^{*}$. D $\psi / D t=R$ with $\psi$. $\nabla \rho^{*} \mathbf{v}=0$, the mathematically equivalent conservation-law form can be written as

$$
\frac{\partial \rho^{*} \Psi}{\partial \mathrm{t}}+\nabla \cdot\left(\rho^{*} \mathbf{v} \psi\right)=\rho^{*} \mathrm{R}
$$

The nonoscillatory forward-in-time algorithm employed to integrate (6) to the secondorder in time and space can be written in a compact functional form as

$$
\psi_{i}^{n+1}=A_{i}\left(\tilde{\Psi}, v^{n+1 / 2}, \rho^{*}\right)+0.5 \delta t R_{i}^{n+1} \equiv \psi_{i}+0.5 \delta t R_{i}^{n+1}
$$

where $\psi_{i}^{n+1}$ is the solution sought at the mesh point $\left(t^{n+1}, x_{i}\right), \tilde{\psi} \equiv \psi^{n}+0.5 \delta R^{n}$, and the reduction of the effective density $\rho^{*}$ is accounted for within the discrete transport operator $\mathbf{A}$ - a second-order-accurate finite-volume nonoscillatory two-time level advection transport algorithm MPDATA, widely documented in the literature (Smolarkiewicz and Szmelter, 2005a,b, 2009; Szmelter and Smolarkiewicz, 2010). ${ }^{2}$ Advecting the auxiliary variable $\tilde{\Psi}$ in (7) - rather than $\psi$ alone and combining $0.5 \delta t\left(R^{n}+R^{n+1}\right)$ outside of $A$, in the spirit of Crank-Nicholson schemes - reproduces the trapezoidal-integral structure of the underlying Lagrangian expression $\mathrm{D} \psi / \mathrm{Dt}=\mathrm{R}$, and compensates for the first-order error $\propto \nabla \cdot \mathbf{v R}$ characteristic of forward-in-time schemes (Smolarkiewicz and Margolin, 1993). Centering in time the advective velocity $\mathbf{v}^{n+1 / 2}$ that appears as an argument of $\mathbf{A}$ is required to compensate for the first order errors $\propto \partial \mathbf{v} / \partial t$ (Smolarkiewicz, 2006). For the second-order accuracy of the solution in (7), it suffices to provide only a first order accurate estimate of $\mathbf{v}_{\mathbf{i}}^{\mathrm{n}+1 / 2}$. The simplest choice is the linear extrapolation from $\mathbf{v}^{\mathrm{n}-1}$ and

\footnotetext{
${ }^{2}$ The crux of the method is the iterative application of the first-order-accurate, yet sign-preserving generic upwind scheme (alias donor cell), with first iteration providing first-order accurate solution, and subsequent iterations compensating for errors of the preceding iterations. In its basic form, the scheme is second-order accurate and sign preserving for arbitrary flows.
} 
$\mathbf{v}^{\mathrm{n}}$ (cf. Smolarkiewicz and Szmelter, 2009); for the soundproof equations this also assures that $\mathbf{v}^{\mathbf{n}+1 / 2}$ satisfies the mass continuity equation, provided $\mathbf{v}^{\mathbf{n}-1}$ and $\mathbf{v}^{\mathrm{n}}$ do. Here, however, we exclusively use the nonlinear predictor

$$
\begin{aligned}
& \mathbf{v}_{i}^{n+1 / 2}=\tilde{\mathbf{v}}_{i}^{n}-0.5 \delta t \mathbf{v}_{i}^{n} \cdot \nabla_{i} \tilde{\mathbf{v}}^{n}-0.5 \delta t \Theta_{i}^{n} \nabla_{i} \phi^{n+1 / 2} \\
& 0=\nabla_{i} \cdot\left(\rho^{*} \mathbf{v}^{n+1 / 2}\right),
\end{aligned}
$$

as it benefits the stability and accuracy of computations on co-located meshes and grids (Smolarkiewicz and Margolin, 1993). In (8), $\tilde{\mathbf{v}}=\mathbf{v}+0.5 \delta \mathrm{tF}$, with $\mathbf{F}$ referring to the cumulative specific force on the rhs of the third equation in (5), and $\nabla_{\mathbf{i}}$ symbolizes a discrete gradient operator (section 3.4); accordingly, the estimate of $\mathbf{v}^{n+1 / 2}$ is congruous with firstorder-accurate Lagrangian trajectory integrals of Dv/Dt $=\mathbf{F}$. The dependent variable $\phi$, in the third term on the rhs of the predictor, is an implicit pressure correction enabling the enforcement of the mass-continuity constraint. It is determined by formulating and solving an elliptic boundary value problem, as discussed below.

\subsection{Boundary value problems}

Elliptic boundary value problems (BVPs) and their efficient solution are at heart of soundproof models. They arise naturally by demanding solenoidal momentum fields dictated by the mass continuity constraints. The auxiliary problem of the advective velocity predictor in (8) is a convenient vehicle to outline the principles of formulating such a BVP. Multiplying the velocity predictor formula in (8) by $\rho_{\mathrm{i}}^{*}$ to form the equivalent momentum predictor, and applying the discrete divergence operator to the resulting formula leads to

$$
\nabla_{\mathbf{i}} \cdot \rho^{*} \mathbf{v}^{\mathrm{n}+1 / 2}=\nabla_{\mathbf{i}} \cdot \rho^{*}\left(\mathbf{v}-\mathrm{C} \nabla \boldsymbol{\phi}^{\mathrm{n}+1 / 2}\right),
$$

where notation has been abridged by denoting the explicit part of the velocity predictor as $\mathbf{v} \equiv \tilde{\mathbf{v}}^{\mathrm{n}}-0.5 \delta \mathrm{t} \mathbf{v}^{\mathrm{n}} \cdot \nabla \tilde{\mathbf{v}}^{\mathrm{n}}$ and the implicit part as $\mathrm{C} \nabla \phi^{\mathrm{n}+1 / 2} \equiv 0.5 \delta \mathrm{t} \Theta^{\mathrm{n}} \nabla \phi^{\mathrm{n}+1 / 2}$. Because $\nabla_{\mathbf{i}} \cdot \rho^{*} \mathbf{v}^{\mathrm{n}+1 / 2}$ must vanish for all mesh points, the discrete Poisson problem implied by (8) is

$$
\forall_{\mathrm{i}} \stackrel{1}{\rho^{*}} \nabla_{\mathrm{i}} \cdot \rho^{*}\left(\mathbf{v}-C \nabla \phi^{\mathrm{n}+1 / 2}\right)=0
$$

where the division by $\rho^{*}$ has been introduced arbitrarily to mitigate problem stiffness and to assure uniform solution accuracy in flows with large density contrasts. The Poisson problem in (10) is subject to the Dirichlet velocity boundary conditions $\mathbf{n} \cdot \mathbf{v}_{\mathrm{B}}^{\mathrm{n}+1 / 2}=\mathbf{n} \cdot \mathbf{v}_{\mathrm{e}}$, which imply Neumann boundary conditions for $\phi, \mathbf{n} \cdot \nabla \phi_{B}^{n+1 / 2}=\mathbf{n} \cdot\left[\left(\mathbf{v}-\mathbf{v}_{\mathrm{e}}\right) / \mathrm{C}\right]_{B}^{\mathrm{n}+1 / 2}$; here subscript ${ }_{B}$ refers to the boundary points, and $\mathbf{n}$ is the outward unit normal to the boundary $\partial \Omega$ of the integration domain $\Omega$. Such a design of the boundary conditions assures the integrability condition ${ }_{\partial \Omega} \rho^{*} \mathbf{n} \cdot \mathbf{v}^{\mathrm{n}+1 / 2}=0$ for (10), given ${ }_{\partial \Omega} \mathbf{n} \cdot \mathbf{v}_{\mathrm{e}}=0$. 
The BVP (10) is optional, ${ }^{3}$ and relatively simple compared to the problem implied by the model algorithm (7). The symbolic relation (7) is used to form a system of discrete equations implicit with respect to all dependent variables in (5), because velocity, pressure, and potential temperature are assumed to be unknown at $n+1$. The general idea of solving such a system, is to first invert it algebraically to build a closed-form expression for the momentum components, and then to apply the discrete divergence operator (consistent with that used in the advection operator $\mathbf{A})^{4}$ to formulate the sparse linear system representing the elliptic equation for $\varphi^{\prime}$ on the mesh. Solution of this sparse system provides an updated pressure variable, such that the resulting velocity field satisfies the discrete mass-continuity constraint to a specified accuracy threshold. The latter, together with the consistency of the divergence operators in mass continuity and advection, prevents generation of spurious fine-scale tendencies in the transport of smooth fields. In the following we illustrate this procedure assuming a 2D subset of the governing pquations in the vertical (xz) plane with a horizontally uniform ambient state $v_{e}(z), \theta_{e}(z)=u_{e}(z), 0, \theta_{e}(z)$, so the respective realizations of (7) for $\mathrm{u}, \mathrm{w}$, and $\theta^{\prime}$ become

$$
\begin{aligned}
& u=\hat{u}-\delta_{h} t \Theta \partial_{x} \varphi^{\prime} \\
& w=\hat{w}-\delta_{h} t \Theta \partial_{z} \varphi^{\prime}+\delta_{h} t \beta \theta^{\prime} \\
& \theta^{\prime}=\hat{\theta}^{\prime}-\delta_{h} t w \partial_{z} \theta_{e} .
\end{aligned}
$$

Here $\delta_{h} t=0.5 \delta \mathrm{t}, \beta=g Y / \bar{\theta}, \partial_{\xi}$ (for $\xi=x$ or $z$ ) is a shorthand for respective spatial partial derivatives, and all references to the mesh location ${ }_{i}^{n+1}$ have been suppressed for conciseness, as there is no ambiguity. Inserting the third equation of (11) into the second, regrouping the terms $\propto \mathrm{w}$ on the left-hand side, and dividing the entire equation by the coefficient $\left[1+\left(\delta_{h} t\right)^{2} \beta \partial_{z} \theta_{e}\right]$ that multiplies $w$, results in the closed form formulae for the update of the velocity components

$$
\begin{aligned}
& u=\hat{u}-c^{x x} \partial_{x} \varphi^{\prime} \\
& w=\hat{w}-c^{z z} \partial_{z} \varphi^{\prime}
\end{aligned}
$$

with the modified explicit parts of the solution

$$
\hat{u}=\hat{u}, \quad \hat{w}=\left(\hat{w}+\delta_{h} t \beta \hat{\theta}^{\prime}\right)\left[1+\left(\delta_{h} t\right)^{2} \beta \partial_{z} \theta_{e}\right]^{-1} ;
$$

and coefficients

$$
C^{x x}=\delta_{h} t \check{\Theta}, \quad C^{z z}=\delta_{h} t \check{\Theta}\left[1+\left(\delta_{h} t\right)^{2} \beta \partial_{z} \theta_{e}\right]^{-1}
$$

where $\odot$ (equal to unity in the anelastic system) marks a suitable approximation of $\Theta=$ $\theta^{n+1} / \theta_{0}$, circumventing the nonlinearity of (11) for the pseudo-incompressible system, to

\footnotetext{
${ }^{3}$ Recall that (8) can be replaced by a linear extrapolation from $\mathbf{v}^{\mathrm{n}-1}$ and $\mathbf{v}^{\mathrm{n}}$.

${ }^{4}$ This is important to assure that advection of a locally constant field reproduces the mass continuity equation.
} 
be discussed later in this section. Having derived (12), the remaining part of formulating the BVP associated with (7) follows the path outlined for (8). Multiplying both equations in (12) by $\rho^{*}$ and applying the discrete divergence $\left(\partial_{x}, \partial_{z}\right) \cdot$ to the resulting momentum vector $\left(\rho^{*} u, \rho^{*} w\right)$ generates the associated Poisson problem

$$
\frac{1}{\rho^{*}}\left[\partial_{x} \rho^{*}\left(\hat{u}-C^{x x} \partial_{x} \varphi^{\prime}\right)+\partial_{z} \rho^{*}\left(\hat{w}-C^{z z} \partial_{z} \varphi^{\prime}\right)^{\prime} \equiv-\left(L \varphi^{\prime}-R\right)=0\right.
$$

which differs from the auxiliary one in (10) by its explicit counterpart $R=\left(\partial_{x} \rho^{*} \hat{u}+\right.$ $\left.\partial_{z} \rho^{*} \hat{w}\right) / \rho^{*}$, and by the coefficients of the linear operator $L=\left(\rho^{*}\right)^{-1}\left(\partial_{x} \rho^{*} C^{x x} \partial_{x}+\partial_{z} \rho^{*} C^{z z} \partial_{z}\right)$. Furthermore, extending (11) to admit the Rayleigh friction (with an inverse time scale of attenuation $\alpha$ ) and the Newtonian cooling (with a corresponding inverse time scale $\alpha$ ) — useful for mitigating wave reflection from rigid boundaries (Davies, 1983; Kosloff and Kosloff, 1986) or for simulating solid bodies immersed in the flow (Smolarkiewicz et al., 2007; Smolarkiewicz and Winter, 2010) —

$$
\begin{aligned}
& u=\hat{u}-\delta_{h} t \Theta \partial_{x} \varphi^{\prime}-\delta_{h} t \alpha\left(u-u_{e}\right) \\
& w=\hat{w}-\delta_{h} t \Theta \partial_{z} \varphi^{\prime}+\delta_{h} t \beta \theta^{\prime}-\delta_{h} t \alpha\left(w-w_{e}\right) \\
& \theta^{\prime}=\hat{\theta}^{\prime}-\delta_{h} t w \partial_{z} \theta_{e}-\delta_{h} t \alpha^{\prime} \theta^{\prime}
\end{aligned}
$$

leads to the analogous Poisson problem to (15), yet with the redefined explicit parts of the solution

$$
\hat{u}=\frac{\hat{u}+\delta_{h} t \alpha u_{e}}{1+\delta_{h} t \alpha}, \quad \hat{w}=\frac{\hat{w}+\delta_{h} t \alpha w_{e}+\delta_{h} t \beta \hat{\theta}^{\prime}\left(1+\delta_{h} t \alpha\right)^{\prime}}{\left(1+\delta_{h} t \alpha\right)+\left(\delta_{h} t\right)^{2} \beta \partial_{z} \theta_{e}\left(1+\delta_{h} t \alpha\right)^{-1}} ;
$$

and accordingly modified coefficients of the linear operator

$$
C^{x x}=\frac{\delta_{h} t \check{\Theta}}{\left(1+\delta_{h} t \alpha\right)}, \quad C^{z z}=\frac{\delta_{h} t \check{\Theta}}{\left(1+\delta_{h} t \alpha\right)+\left(\delta_{h} t\right)^{2} \beta \partial_{z} \theta_{e}\left(1+\delta_{h} t \alpha^{\prime}\right)^{-1}} .
$$

Notably, the closed-form formula for $\theta^{\prime}$ employed to derive (12), (13) and (14) is that in (11). However, for the extended case (16), an intermediate step involves elementary inversion of the third equation in set (16), resulting in

$$
\theta^{\prime}=\frac{\hat{\theta}^{\prime}-\delta_{h} t w \partial_{z} \theta_{e}}{1+\delta_{h} t \alpha}
$$

to be subsequently inserted in the second equation of (16)

The three examples of formulating BVPs, dictated by the model, gradually increase the level of difficulty, starting with a vector notation in (8) and then resorting to a component notation in (11) and (16) to better convey involved details. As can be seen from the 
increasing complexity of the presentation, the component notation quickly becomes impractical. In EULAG literature, the design of the associated BVPs is typically reduced to the vector/operator symbolism, for the sake of conciseness. The details of a full 3D Poisson problem associated with the governing equations cast in generalized time-dependent curvilinear coordinates - with implicit representation of the buoyancy, Coriolis, and attenuation forcings on the right-hand-sides, and assuming 3D ambient states - are presented using tensor notation in Prusa and Smolarkiewicz (2003). The formulae therein for the explicit components of the solution and for the coefficients of the linear operator are independent of spatial discretization, and rely on the algorithmic construct akin to the one presented here. Thus, for unstructured meshes they can be used as a reference, given a co-located data structure for all prognosed dependent variables.

The prognostic model algorithm (7) does not distinguish between the anelastic or the pseudo-incompressible equation sets. However, there is a significant difference when it comes to formulating the BVP. The coefficient $\Theta$ in front of the pressure gradient is fixed in the anelastic model, whereas it is a function of dependent fluid variable $\theta$ in the pseudoincompressible model. Due to nonlinearity of the pressure gradient term in (2), the algorithm (16) is executed iteratively

$$
\begin{aligned}
& u^{v}=\hat{u}-\delta_{h} t \Theta^{v-1} \partial_{x} \varphi^{\prime v}-\delta_{h} t \alpha\left(u^{v}-u_{e}\right) \\
& w^{v}=\hat{w}-\delta_{h} t \Theta^{v-1} \partial_{z} \varphi^{\prime v}+\delta_{h} t \beta \theta^{\prime v}-\delta_{h} t \alpha\left(w^{v}-w_{e}\right) \\
& \theta^{\prime v}=\hat{\theta}-\delta_{h} t w^{v} \partial_{z} \theta_{e}-\delta_{h} t \alpha \theta^{\prime v},
\end{aligned}
$$

where $v=1, . ., m$ numbers the iterations, and at each iteration the linear elliptic problem (15), (17) and (18) is solved with the lagging $\odot=\Theta^{v-1}$. The first guess $\Theta^{0}$ predicted using the non-perturbation form of the entropy equation $D \theta / D t=-\alpha\left(\theta-\theta_{e}\right)$ already suffices for second-order accuracy. Further iterations converge rapidly, while adding little overhead compared to the anelastic solver. In practice, there is no gain in using $\mathrm{m}>$ 2; for example, in the global baroclinic instability simulations in Smolarkiewicz (2011),

$\theta^{\prime} v-\theta^{\prime(v-1)} \quad \infty^{\sim} \mathrm{O}\left(10^{-4}\right) \mathrm{K}$ and $\sim \mathrm{O}\left(10^{-6}\right) \mathrm{K}$ for $\mathrm{m}=2$ and $\mathrm{m}=3$. Because the solution of the elliptic problem uses an iterative solver with a physically motivated accuracy-based stopping criterion $\left(\delta \mathrm{t} / \rho^{*}\right) \nabla \cdot\left(\rho^{*} \mathbf{v}\right) \quad \infty<\varepsilon$ (Smolarkiewicz et al., 1997), ${ }^{5}$ the work within the solver decreases dramatically past $v=1$.

\subsection{Highlights of the elliptic solver}

Among the most effective methods reported for solving difficult elliptic problems, such as those arising in simulation of atmospheric circulations, are preconditioned nonsymmetric Krylov-subspace - viz. conjugate-gradient (CG) type — iterative schemes. Like

\footnotetext{
${ }^{5}$ This stopping criterion relates dimensionless flow divergence and magnitude - i.e. the divergence and magnitude of the Courant number $\mathrm{C} \sim \mathrm{O}(1)$ - typical values of $\varepsilon$ are $10^{-5} \mathrm{C}$.
} 
the classical stationary Richardson and Frankel (alias second-order Richardson) schemes (Birkhoff and Lynch, 1984, chpt. 5) they effectively generate an approximate solution of the governing elliptic problem, say (15), recurrently by linearly combining the initial guess $\varphi^{\prime}{ }^{0}$ with the subsequent iterates of the initial residual error, $r^{0}=L\left(\varphi^{0}\right)-R$, under the linear operator $\mathbf{L}$, such that

$$
\forall_{i} \quad \varphi_{i}^{\prime}=\varphi_{i}^{0}+\nabla_{\gamma=1}^{v} a_{v, \gamma} L_{i}^{\gamma-1}\left(r^{0}\right)
$$

However unlike the stationary schemes, which predetermine the coefficients $a_{v, y}$ based on spectral properties of the operator $L$, the CG methods rely on variational principles to determine coefficients optimal for the iteration convergence, by minimizing error norms of the approximate solutions (cf., sections 11 and 12 in Axelsson, 1994). While there exist a number of optional nonsymmetric Krylov solvers common in computational research and engineering (Axelsson, 1994; Saad, 1995; Greenbaum, 2002), our method of choice is the restarted generalized conjugate residual GCR(k) algorithm of Eisenstat et al. (1983), proven successful in geophysical and astrophysical applications (Prusa et al., 2008; Ghizaru et al., 2010, and references therein). Although the GCR scheme is widely documented in the literature, here we summarize it briefly for the reader's convenience, and for further reference in section 4 as well as in the accompanying paper (Piotrowski et al., 2011, ibid.) devoted to massive parallelization of soundproof models.

The mathematical formalism of CG algorithms typically refers to methods of linear algebra for solving large sparse linear systems, thus minimizing error norms of their approximate solutions over Krylov (vector) subspaces spanned by the subsequent iterates of the residual error vector $\mathbf{r}^{0}=\left(r_{1}^{0}, r_{2}^{0}, \ldots, r_{m}^{0}\right)$ under the sparse $\mathrm{m} \times \mathrm{m}$ matrix $\mathbf{L}$ representing the differential operator $L$ on the mesh of $m$ points. However, an alternative interpretation in terms of discrete integrations of the pseudo-time augmented PDEs (cf. Smolarkiewicz and Margolin, 2000, and references therein) may be more appealing to a physicist. Just like the first- and second-order Richardson methods may be viewed as discrete integrations of the heat and damped oscillation equations (Birkhoff and Lynch, 1984, sections 4.9 and 4.15, respectively), the preconditioned GCR $(\mathrm{k})$ algorithm can be derived ${ }^{6}$ by augmenting BVPs of the preceding section with a kth-order damped oscillation equation

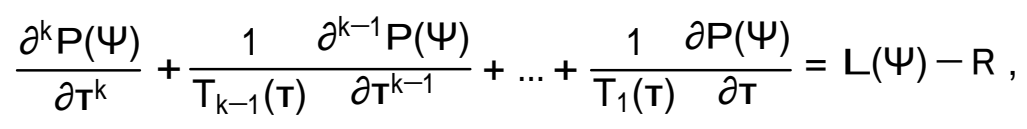

where $\Psi$ encompasses $\phi$ and $\varphi^{\prime}$ that appear in (10) and (15), respectively, and the operator $P$ is the so-called preconditioner (left), to be discussed shortly. Discretizing (21) in a pseudo-time $\mathbf{T}$, to form the affine discrete equation for the progression of the residual errors

${ }^{6}$ For a complete derivation of the basic CGR(1) algorithm see Smolarkiewicz and Margolin (1994). 
$r=L(\Psi)-R$; and determining the optimal parameters $T_{1}, . ., T_{k-1}$ and the increment $\Delta \mathrm{T}$ (all variable in $\mathrm{T}$ ), to assure minimization of the residual errors progressively over $=1,2, . ., \mathrm{k}$ iterations in the norm defined by the inner product $(r r) \equiv{ }_{\forall i} r_{i} r_{i}$, leads to the following algorithm.

For any initial guess $\Psi_{i}^{0}$, set $r_{i}^{0}=L_{i}\left(\Psi^{0}\right)-R_{i}, q_{i}^{0}=P_{i}^{-1}\left(r^{0}\right)$; then iterate:

$$
\begin{aligned}
& \text { For } n=1,2, \ldots \text { until convergence do } \\
& \text { for } v=0, . ., k-1 \text { do } \\
& \beta=-\frac{\left(r^{\vee} L\left(q^{v}\right)\right)}{\left(L\left(q^{v}\right) L\left(q^{v}\right)\right)}, \\
& \Psi_{i}^{v+1}=\Psi_{i}^{v}+\beta q_{i}^{v}, \\
& r_{i}^{v+1}=r_{i}^{v}+\beta L_{i}\left(q^{v}\right) \text {, } \\
& \text { exit if } r^{v+1} \leq \varepsilon^{*} \text {, } \\
& e_{i}=P_{i}^{-1}\left(r^{v+1}\right) \text {, } \\
& \forall_{\mathrm{i}} \text { evaluate } \mathrm{L}_{\mathrm{i}}(\mathrm{e})=\frac{1}{\rho^{*}} \nabla_{\mathrm{i}} \cdot \mathbf{C} \nabla \mathrm{e}, \\
& \forall_{\mathrm{l}=0, v} \quad \alpha_{\mathrm{l}}=-\frac{\left(\mathrm{L}(e) L\left(q^{\prime}\right)\right)}{\left(L\left(q^{\prime}\right) L\left(q^{\prime}\right)\right)}, \\
& q_{i}^{v+1}=e_{i}+>_{l=0} \alpha_{1} q_{i}^{l}, \\
& \mathrm{~L}_{\mathrm{i}}\left(\mathrm{q}^{\mathrm{v}+1}\right)=\mathrm{L}_{\mathrm{i}}(\mathrm{e})+\prod_{\mathrm{l}=0} \mathrm{a}_{\mathrm{l}} \mathrm{L}_{\mathrm{i}}\left(\mathrm{q}^{\mathrm{l}}\right) \\
& \text { end do , } \\
& \text { reset }[\Psi, r, q, L(q)]_{i}^{k} \text { to }[\Psi, r, q, L(q)]_{i}^{0} \text {, } \\
& \text { end do . }
\end{aligned}
$$

For convergence, the GCR(k) algorithm above requires LP $^{-1}$ to be negative definite ${ }^{7}$ but not necessarily self-adjoint ${ }^{8}$. Direct evaluation of the elliptic operator on the mesh symbolized above as $\mathbf{L}=\left(\rho^{*}\right)^{-1} \nabla \cdot \rho^{*} \mathbf{C} \nabla$ with $\mathbf{C}$ referring to the coefficient matrix, e.g.,

\footnotetext{
${ }^{7}$ An operator $L$ is said to be definite if $(\xi\llcorner(\xi))$ is either strictly positive (positive definite) or strictly negative (negative definite) for all $\xi$.

${ }^{8}$ An operator $L$ is said to be self-adjoint if $(\xi L(\zeta))=(\zeta L(\xi))$ for all $\xi$ and $\zeta$.
} 
the diagonal matrix with entries $C^{x x}$ and $C^{z z}$ in (18) - takes place only once per iteration following the preconditioning $\mathrm{e}=\mathrm{P}^{-1}\left(\mathrm{r}^{\mathrm{v}+1}\right)$ that provides an estimate of the solution error $e^{v+1}=\Psi^{v+1}-\Psi_{\text {exact }}$. For consistency with the physically-motivated stopping criterion mentioned at the end of the preceding section, $\varepsilon^{*}=\varepsilon / \delta$ t in step 4 of the inner loop of the GCR(k) algorithm.

The preconditioning operator $\mathrm{P}$ is important. Its role is to substitute the governing elliptic problem $L(\Psi)-R=0$ with an auxiliary problem $P^{-1}(L(\Psi)-R)=0$ that converges faster than the original problem due to a better conditioning (viz. a lesser stiffness) of the auxiliary elliptic operator $\mathrm{P}^{-1} \mathrm{~L}$. There is no general method for designing an optimal preconditioner (Axelsson, 1994, section 7). For the preconditioner to be useful, the convergence of the augmented (preconditioned) problem must be sufficiently rapid to overcome the effort associated with "inverting" the preconditioner itself (i.e., computing $\mathrm{P}^{-1}(\cdot)$ ). In general, the closer the preconditioner approximates the original operator $L$, the faster the solver converges but the more difficult it is to compute $P^{-1}(\cdot)$. Depending on the intricacy of the problem, $\mathrm{P}$ can be as simple as an identity operator (viz. no preconditioning), or as complex as $\mathbf{L}$ itself (resulting then in a direct solver providing the precise solution in a single GCR iteration (Smolarkiewicz et al., 2004). Calculations of deep gravity waves in section 4 exemplify the flexibility of preconditioning and its importance for efficient solutions of associated BVPs.

\subsection{Highlights of spatial discretization}

The derivations and details of median-dual unstructured mesh discretization of differential operators entering MPDATA schemes, symbolized with A in (7), are provided in Smolarkiewicz and Szmelter (2005a,b) and Szmelter and Smolarkiewicz (2006). For completeness, here we highlight a key tool for designing discrete differential operators for edge-based control-volume schemes.

Figure 1 marks a face $S_{j}$ of an arbitrary computational cell containing vertex $i$, together with the edge connecting vertex $i$ with one of its immediate neighbors $j$; there are I(i) edges connecting the vertex $i$ with its neighbors. $S_{j}$ refers both to the face per se and its surface area. For a differentiable vector field $\mathbf{A}$, the Gauss divergence theorem -

${ }_{\Omega} \nabla \cdot \mathbf{A}={ }_{\partial \Omega} \mathbf{A} \cdot \mathbf{n}$ - applied over the control volume $\mathbf{V}_{\mathbf{i}}$ surrounding vertex $i$ leads to

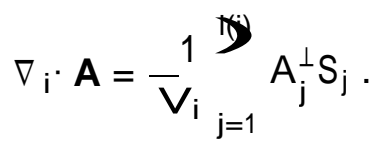

Equation (22) is exact given $\nabla_{\mathbf{i}} \cdot \mathbf{A}$ is interpreted as the mean value of $\nabla \cdot \mathbf{A}$ within the volume $V_{i}$, while $A_{j}^{\perp}$ is interpreted as the mean normal component of the vector $\mathbf{A}$ at the cell face $S_{j}$. The approximation begins with specifying $A_{j}^{\perp}$ in terms of data available on the 
mesh; i.e., in terms of mean values of the field within the control volumes $\mathbf{V}_{\mathbf{i}}$ and $\mathbf{V}_{\mathbf{j}}$. One elementary example is a correspondent of the centered finite-difference approximation on a regular grid

$$
A_{j}^{\perp}=0.5 n_{j} \cdot\left[A_{i}+A_{j}\right],
$$

where $\mathbf{n}_{\mathrm{j}}$ is a mean outward unit normal to the face $S_{j}$. Partial derivatives $\partial \Phi / \partial x^{1}$ of a scalar field $\Phi$ with subscript $\mathrm{I}=1,2$ or 3 referring to Cartesian coordinates $\mathrm{x}, \mathrm{y}$ or $\mathrm{z}$, respectively, can also be interpreted in terms of the Gauss theorem, by representing the derivative as the divergence of the augmented vector field $\Phi \nabla x^{\prime}$; e.g.;

$$
{\frac{\partial \Phi}{\partial x^{l}}}_{i}=\frac{1}{V_{i}}{ }_{j=1} 0.5\left(\Phi_{i}+\Phi_{j}\right) S_{j}^{l}
$$

where $S_{j}^{l}$ denotes the Ith component of the oriented surface element $\mathbf{S}_{\mathbf{j}}=\mathbf{S}_{\mathbf{j}} \mathbf{n}_{\mathbf{j}}$ of the face at the jth edge.

\section{RESULTS: BREAKING OF STRATOSPHERIC GRAVITY WAVE}

To illustrate the capability of unstructured meshes for simulation of multiscale internal gravity wave phenomena, we document the performance of the model presented in the preceding section using an intricate example of non-Boussinesq amplification and breakdown of a deep stratospheric gravity wave. More elementary benchmarks of a weakly and strongly nonlinear Boussinesq flow past an isolated hill were presented already in Szmelter and Smolarkiewicz (2011), using an earlier, explicit version of the model. The current model reproduces those results (not shown). The problem selected here adapts the one originated in Smolarkiewicz and Margolin (1997) and inspired by a study of gravity wave breaking at mesopausal altitudes (Prusa et al., 1996). A small amplitude wave packet, excited by a hypothetical squall line with the top impinging upon the tropopause, propagates into the stratosphere. Because density of the media decreases with altitude, the amplitude of the wave increases with height in proportion to $\bar{\rho}^{-1 / 2}$. When the wave amplitude becomes comparable with the vertical wavelength, the problem becomes inherently nonlinear. Then, the wave overturns and breaks generating bursts of turbulence far from the excitation region. The problem is numerically challenging, because it covers about nine density height scales and vertical wavelengths, and a transition from the linear-wave regime near the bottom of the domain to a vigorous turbulent flow with a broad range of scales about $30 \mathrm{~km}$ aloft. For reference, the evolution of the anelastic solution generated with the structured-grid EULAG model is highlighted in Fig. 2.

The present setup assumes an isothermal stratosphere, with temperature $\mathrm{T}_{0}=222.65 \mathrm{~K}^{\circ}$, and uniform potential temperature stratification $S_{\theta}=d \ln \bar{\theta} / \mathrm{dz}=4.4 \cdot 10^{-5} \mathrm{~m}^{-1}$. The background density decreases exponentially, such that $S_{\rho}=-d \ln \bar{\rho} / \mathrm{dz}=1.535 \cdot 10^{-4} \mathrm{~m}^{-1}$, 

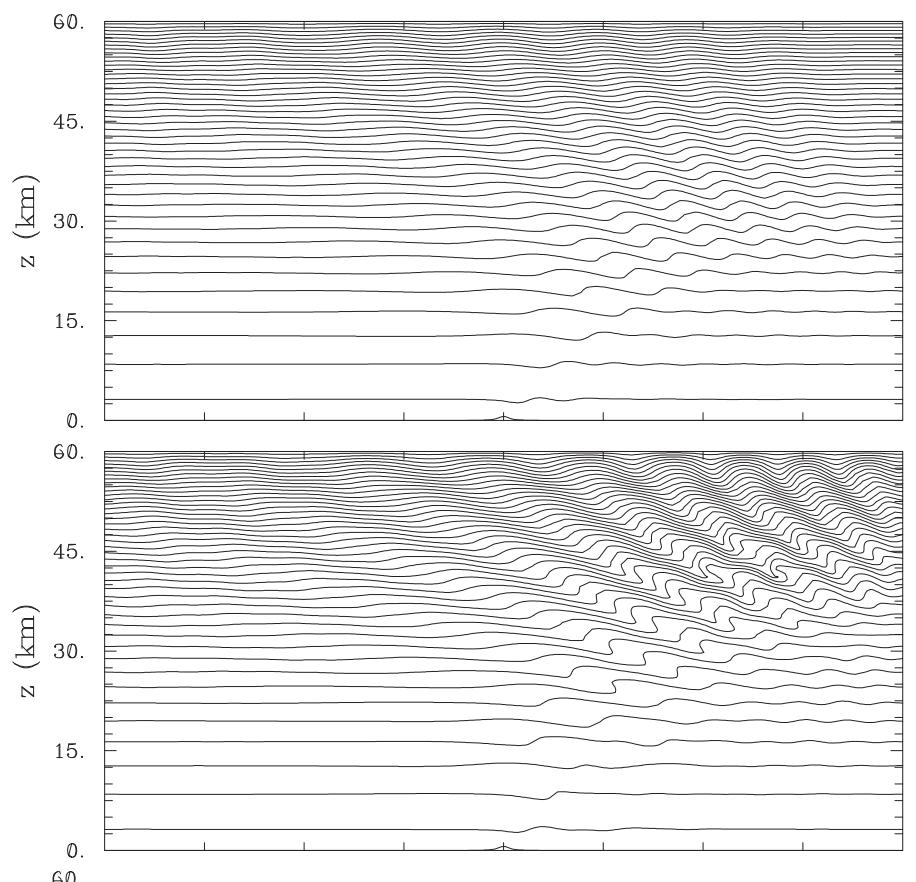

45. 


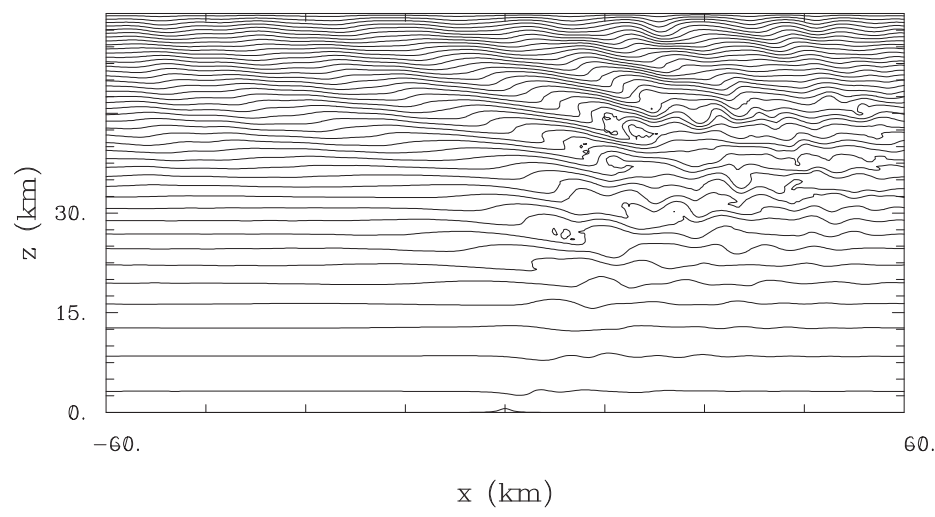

Figure 2: Evolution of isentropes simulated with anelastic equations in the structured-grid EULAG model; from the top to bottom simulated time $t=60$, 90, and $120 \mathrm{~min}$.

so the corresponding density scale $H_{\rho}=1 / S_{\rho}=6515 \mathrm{~m}$ is 3.5 times smaller than the potential temperature scale $H_{\theta}=1 / S_{\theta}$. The ambient wind $\mathbf{v}_{e}=\left(u_{e}, 0\right)$ is constant, with speed $\mathrm{u}_{\mathrm{e}}=\mathrm{U}=20 \mathrm{~ms}^{-1}$, and ambient profile of potential temperature $\theta_{\mathrm{e}}(\mathrm{z})=\bar{\theta}$. The model domain is $60 \mathrm{~km}$ deep, and $120 \mathrm{~km}$ wide. The wave is excited by a small hill embedded in the ambient flow, with the height profile $h(x)=h_{0}\left[1+(x / L)^{2}\right]^{-1}$ centered at the origin of the $[-60 \mathrm{~L}, 60 \mathrm{~L}] \times[0,60 \mathrm{~L}](\mathrm{x}, \mathrm{z})$-domain; the hill's height and half-width are, respectively, 
$\mathrm{h}_{0}=628.319 \mathrm{~m}$ and $\mathrm{L}=1000 \mathrm{~m}$. The problem is inherently nonhydrostatic because $\mathrm{NL} / \mathrm{U} \approx 1$, or, in other words, the dominant horizontal wavenumber of the problem, $1 / \mathrm{L}$, equals the asymptotic wavenumber $\mathrm{N} / \mathrm{U}$ of the induced mountain wave; $\mathrm{Tere} \mathrm{N}=-\overline{\mathrm{gS}_{\theta}}$ denotes the buoyancy frequency. Furthermore, the problem is only weaky nonlinear (the Froude number $\mathrm{Fr}=\mathrm{U} / \mathrm{Nh}_{0} \approx 1.6$ ) with respect to the linear Boussinesq theory (Smith, 1979). Notably, the selected stratification $S_{\theta}$ is four times larger than in Smolarkiewicz and Margolin (1997), to better represent the realism of the stratosphere. Increasing ambient wind $U$ twofold permits other conditions of the experiment in Smolarkiewicz and Margolin (1997) to be retained, except for the rate of the wave development, which is now twice as fast due to the group velocity scaling $\mathrm{cg}_{z} \sim \mathrm{U}^{2} / \mathrm{NL}$. Consequently, the inverse time scales of the gravity-wave absorbers adjacent to the lateral and upper boundaries (section 3.2) change linearly from $\alpha=\alpha=0$ at the absorber edge $20 \mathrm{~km}$ away from a boundary to $\alpha=\alpha=1 / 300 \mathrm{~s}^{-1}$ at the boundary; the time step is set to $\delta \mathrm{t}=5 \mathrm{~s}$; and the onset of wave breaking in the upper half of the model domain, Fig. 2, is observed after half the simulated time of Smolarkiewicz and Margolin (1997), i.e., after 90 min. (as opposed to $180 \mathrm{~min}$.).
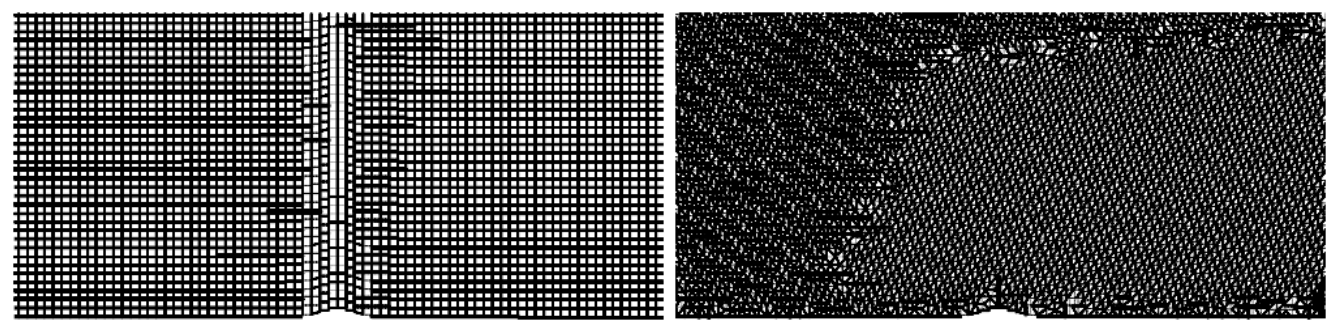

Figure 3: Meshes used in a simulations of a deep stratospheric gravity waves.

Figure 3 shows two different meshes with similar number of points, $59 \cdot 10^{3}$, used in simulations with the edge-based model. On the left, the unstructured mesh, generated explicitly in physical space, mimics the structured grid resulting from the standard terrainfollowing coordinate transformation. Thus, it corresponds to a geometric visualization of products of the uniform grid increment $\delta z=377.36 \mathrm{~m}$ and appropriate metric coefficients employed in the EULAG simulation highlighted in Fig. $2 .{ }^{9}$ On the right, a fully unstructured triangular edge mesh is displayed; cf. Fig. 1. Only mesh portions in the hill proximity are shown. The corresponding solutions at the onset of breaking ( $t=90 \mathrm{~min}$ ) are shown in Figs. 4 and 5. The graphics package used for the edge-based code is different than that employed in Fig. 2. Regardless, the results in Figs. 2, 4 and 5 convey the same underlying physics while deviating only in details. Furthermore, the differences between the pseudo-

${ }^{9}$ EULAG calculations employ a uniform grid with $320 \times 160$ data points, but the governing equations are modified in effect of the coordinate transformation (Prusa and Smolarkiewicz, 2003). 

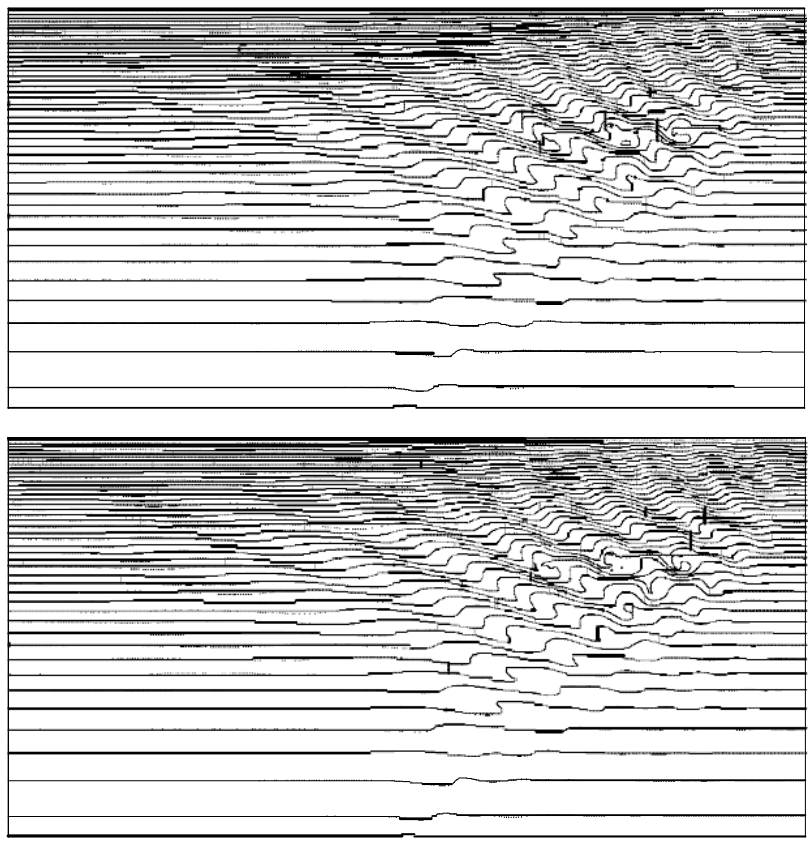

Figure 4: Isentropes at $\mathrm{t}=90 \mathrm{~min}$ simulated with the edge-based model using a regular grid corresponding to EULAG simulations in terrain-following coordinates; the top and bottom panel show solutions of the pseudo-incompressible Durran and anelastic LippsHemler equations, respectively.

incompressible and anelastic solutions in Figs. 4 and 5 appear to be of the same magnitude as the differences between the solutions to the corresponding equations integrated on the regular and unstructured meshes shown in Fig. 3. In other words, the differences between the solutions to the two equations sets are at the level of higher-order truncation errors. The latter is corroborated with the EULAG simulation (not shown), evincing a solution of the pseudo-incompressible equations that is hardly distinguishable from the anelastic result in Fig. 2. This is consistent with the results reported in Smolarkiewicz and Dörnbrack (2008).

In recent theoretical work (Achatz et al., 2010), the authors have shown that the pseudo-incompressible equations are better suited for the study of nonhydrostatic internal gravity wave dynamics than the anelastic equations, because of their asymptotic consistency with fully compressible Euler equations for arbitrary background stratifications. In contrast, the anelastic equations are formally consistent (with the Euler equations) only for a sufficiently weak stratification; i.e., for the temperature height scale $\mathrm{H}_{\theta}$ much larger than the density height scale $\mathrm{H}_{\rho}$. Here, the calculations summarized so far employ real- 

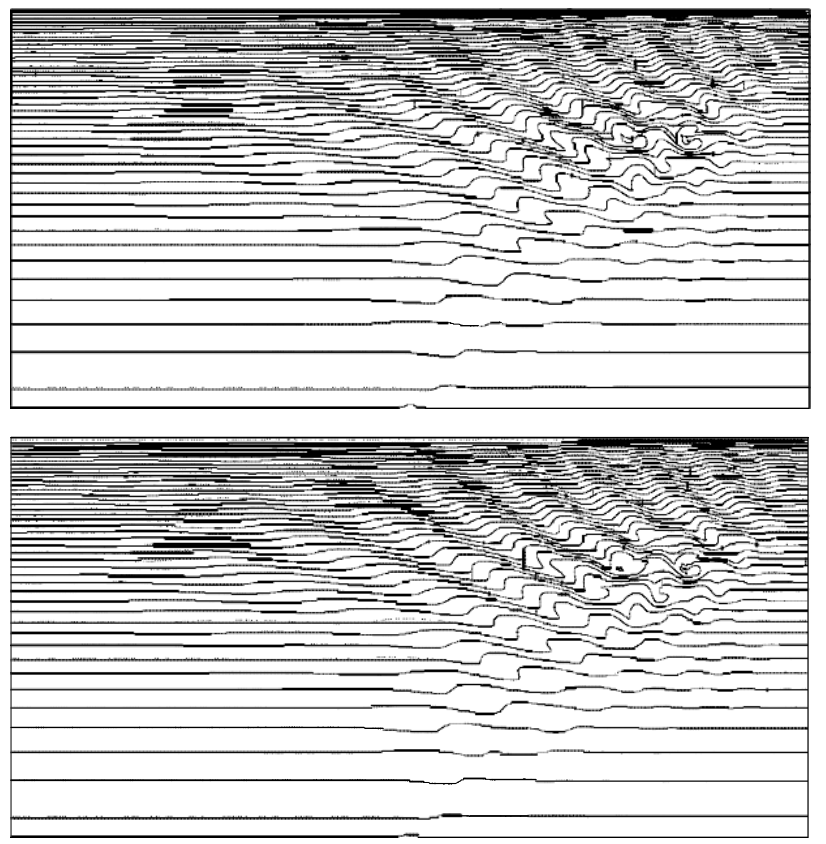

Figure 5: Isentropes at $\mathrm{t}=90 \mathrm{~min}$ simulated with the edge-based model using the unstructured mesh shown in the right panel of Fig. 3; the top and bottom panel display the solutions of the pseudo-incompressible and anelastic equations, respectively.

istic stratospheric conditions, yet they fall in an intermediate regime with $\mathrm{H}_{\theta}$ only a few times larger than $\mathrm{H}_{\rho}$. Although such conditions already violate the assumptions assuring the asymptotic consistency of the anelastic equations (Achatz et al., 2010, section 4.2), the accompanying effects are subtle and not easily detectable in our numerical realizations. To qualify the disparity between the results from the two sets of soundproof equations, we now quadruple the potential temperature stratification $\left(S_{\theta}=17.6 \cdot 10^{-5}\right)$ and double the background wind $\left(U=40 \mathrm{~ms}^{-1}\right)$ to retain the same dominant wavelength and Froude number. Since the group velocity $\mathrm{cg}_{z}$ doubles, the inverse time scale of the gravity wave absorbers is increased to $\alpha=\alpha=1 / 150 \mathrm{~s}^{-1}$, the time step is set to $\delta t=2.5 \mathrm{~s}$, and the onset of wave breaking is now observed at $t=45 \mathrm{~min}$. With the present arrangements, $H_{\theta} \leqslant H_{\rho}$, corresponds to a gas with $R / c_{p} \approx 1.15$. For reference, the EULAG solutions are shown in Fig. 6; the isolines of $\ln \theta$ are displayed to avoid isentrope overcrowding in the upper $\sim 30 \%$ of the model vertical domain. The corresponding solutions on the unstructured triangular mesh are shown in Fig. 7. With close potential temperature and density scale heights, the departures between the two solutions become noticeable: the anelastic equations seem to overpredict the growth rate of the wave field instability. 


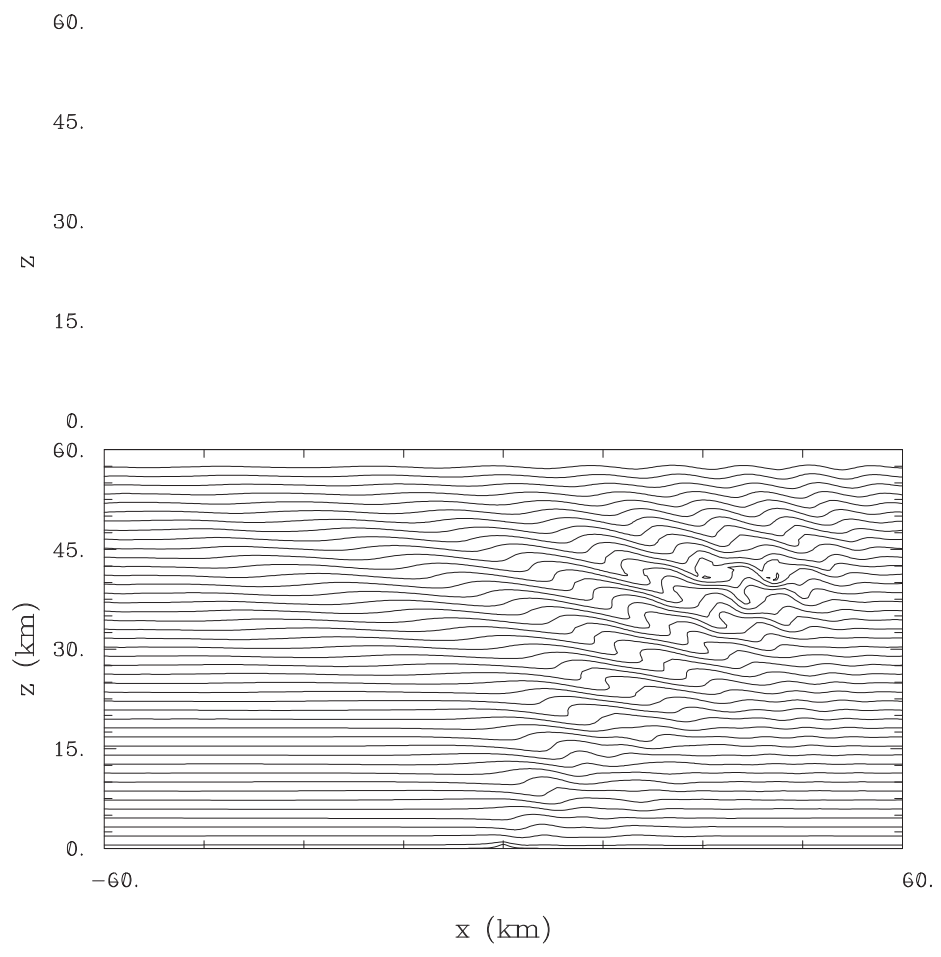

Figure 6: EULAG solution for the potential temperature stratification quadruple of that in Figs. 2, 4 and 5; isolines of $\ln \theta$ are shown at the onset of breaking at $t=45 \mathrm{~min}$ for the pseudo-incompressible and anelastic equations in the top and bottom panel, respectively.

Because two different codes with substantially different spatial discretizations appear to reproduce consistent solution behavior for the two equation sets, we are inclined to believe that this result is robust. For further substantiation, the calculations have been repeated with the semi-Lagrangian option of EULAG, as the latter algorithm tends to mitigate wave overturning for otherwise equally accurate solutions (Smolarkiewicz and Margolin, 1997). Indeed, the semi-Lagrangian results tend to diminish the magnitude of the difference observed in finite-volume solutions, but still show a hint of the more vigorous overturning in the anelastic result. Table 1 quantifies the intensity of the simulated wave overturning with the statistics of the vorticity field $\omega=\delta t\left(\partial_{z} u-\partial_{x} w\right)$ (dimensionless for easy reference to the Courant number $\leqslant 1$ ). The values listed in the table correspond to the results in Figs. 6, 7 and the semi-Lagrangian EULAG runs. ${ }^{10}$ Of particular interest is the

\footnotetext{
${ }^{10}$ Acronyms PSI and ANL refer to the psuedo-incompressible and anelastic equations, abbreviations $\mathrm{CV} /$ grid and SL/grid denote the flux-form and semi-Lagrangian EULAG integrations on the structured grid using terrain-following coordinate transformation, and $\mathrm{CV} /$ mesh marks the control-volume edge-based inte-
} 

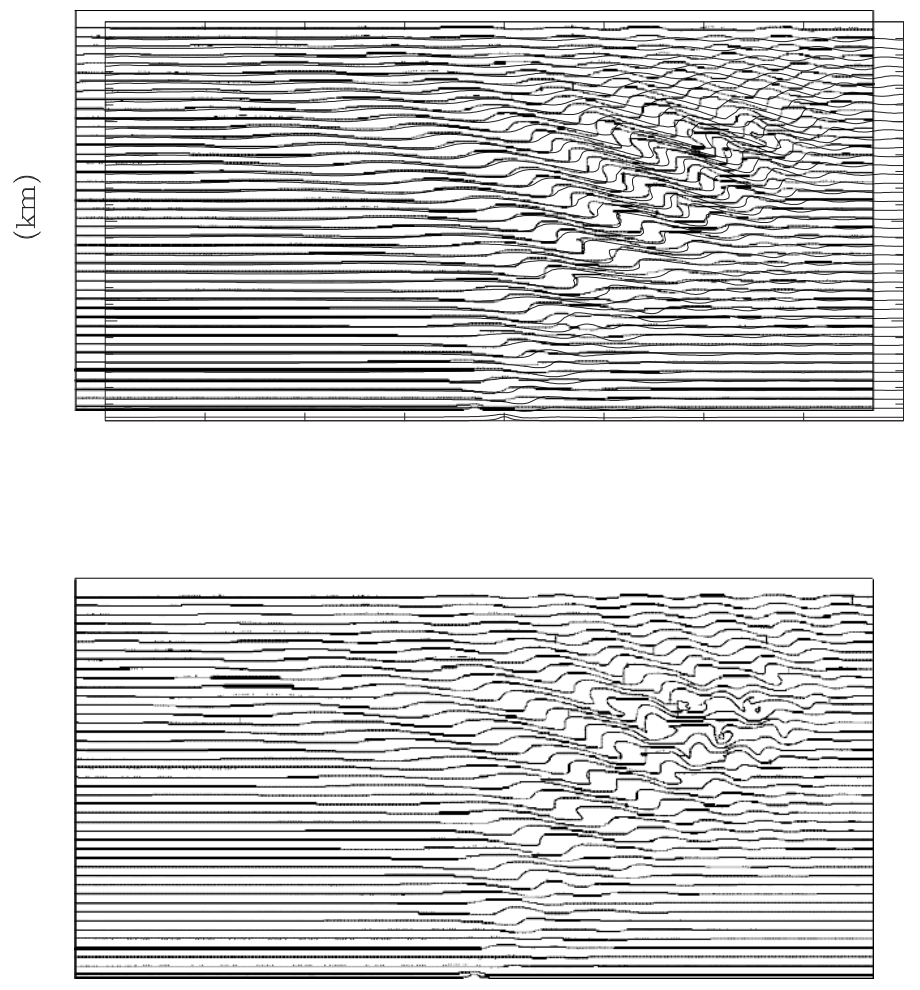

Figure 7: As in Fig. 6, but using the edge-based code with the triangular mesh shown in the right panel of Fig. 3.

$30 \%-50 \%$ contrast between the minima and maxima of $\omega$ in the anelastic simulations, corresponding to enhanced vortical motion in overturning wave crests and strong wind shear in adjacent troughs where isentropes collapse. This contrast is concomitant with the onset of vigorous wave overturning, manifested in vorticity plots with concentrated eddies (not shown).

Table 1: Normalized vorticity: maximum, minimum, mean and standard deviation.

\begin{tabular}{cccccc}
\hline \hline eqs. & numerics & $\max (\omega)$ & $\min (\omega)$ & $\bar{\omega}$ & ${\overline{\left(\omega-\bar{\omega}^{2}\right.}}^{1 / 2}$ \\
PSI & CV/grid & 0.17 & -0.21 & $1.6 \cdot 10^{-4}$ & $3.3 \cdot 10^{-2}$ \\
ANL & CV/grid & 0.27 & -0.41 & $6.4 \cdot 10^{-5}$ & $3.5 \cdot 10^{-2}$ \\
PSI & CV/mesh & 0.28 & -0.24 & $2.0 \cdot 10^{-4}$ & $3.7 \cdot 10^{-2}$ \\
ANL & CV/mesh & 0.24 & -0.36 & $9.5 \cdot 10^{-5}$ & $3.6 \cdot 10^{-2}$ \\
PSI & SL/grid & 0.28 & -0.30 & $2.1 \cdot 10^{-4}$ & $3.1 \cdot 10^{-2}$ \\
ANL & SL/grid & 0.18 & -0.24 & $7.2 \cdot 10^{-5}$ & $3.0 \cdot 10^{-2}$ \\
\hline \hline
\end{tabular}

grations on the triangular mesh. 
Notwithstanding the robustness of the result illustrating the solution departures for the two sets of soundproof equations, its physical significance appears minor. First, the adopted conditions of roughly equal density and potential temperature height scales are unrealistically extreme. Second, as illustrated in Fig. 2, the problem is transient. After the waves break and transform the upper portion of the main wave train into a field of turbulence (like in the lowest panel of Fig. 2), the two solutions again become hard to distinguish (not shown). The latter suggests that the solution departures in Figs. 6 and 7 at the onset of breaking merely indicate that the pseudo-incompressible result is somewhat delayed compared to the anelastic solution. This by no means downplays the importance of the pseudo-incompressible equations. To the contrary, our results document that in the class of flow problems addressed these equations are useful to assess the accuracy of anelastic solutions compared to hypothetical solutions of compressible Euler equations.

We end this section with a comment on the relative computational efficacy of the two sets of soundproof equations in the structured-grid and the edge based unstructured mesh model. The selected gravity wave problem is stiff, so the efficacy of any soundproof model strongly depends on the performance of the elliptic pressure solver. In the structured grid model EULAG the elliptic pressure equation is far more complex than that in (15), (17) and (18), due to the terrain-following coordinate transformation adding cross-derivative terms $\partial_{x z} \varphi^{\prime}$ and metric coefficients to the linear operator $L$ (Prusa and Smolarkiewicz, 2003). On the other hand, EULAG employs a powerful preconditioner, with $P$ closely approximating $L$ by merely removing the cross-derivative terms. The estimation of the solution error (step 5 in the inner loop of the GCR(k) algorithm in section 3.3) then employs a few iterations of a stationary Richardson-type scheme with a direct (tri-diagonal type) inversion in the vertical (Smolarkiewicz et al., 2004), thus eliminating the essential stiffness of the problem. The resulting solver converges (for the problem studied) to the specified accuracy threshold $\varepsilon=10^{-5} \mathrm{C}$ (cf. footnote 4) with no more than 10 evaluations of the generalized Laplacian (step 6 in the inner loop of the GCR(k) solver) per the pseudoincompressible model time step (on average over 1440 time steps) and even less for the anelastic model; see also (Prusa et al., 2001) for a discussion of the solver convergence in function of the solution evolution. In the unstructured mesh model such a preconditioner is unavailable. The unstructured mesh model calculations discussed in this paper employed a simple diagonal preconditioner $\mathrm{P}^{-1}=1 / \Theta{ }^{11}$ which assured the solver convergence to the same accuracy threshold, with no more than 42 and 68 evaluations of the generalized Laplacian per model time step (on average) for the anelastic and pseudo-incompressible equations respectively. However, this does not imply a proportionally larger expense of the solver in the unstructured mesh model, because the preconditioner and the linear operator are both simpler than their EULAG counterparts. In terms of a raw wallclock time, evalu-

\footnotetext{
${ }^{11}$ Alternatively, the division by $\rho^{*}$ introduced in (10) may also be interpreted as a diagonal preconditioning of $\nabla_{i} \cdot \rho^{*}\left(\mathbf{v}-C \nabla \phi^{n+1 / 2}\right)=0$, thus resulting in a composite diagonal preconditioner $P^{-1}=1 /\left(\rho^{*} \Theta\right)$ of the generic linear operator $L=\nabla \cdot \rho^{*} C \nabla$ in the GCR(k).
} 
ating the unstructured mesh solutions for the extreme stratification took 12 and 15 minutes of a single processor Dell Precision 690n workstation, respectively, for the anelastic and pseudo-incompressible model. The wallclock time of the EULAG calculations was minuscule ( $\leqslant 1 \mathrm{~min}$ ), but these runs employed 32 processors of IBM Power 575 supercomputer. This shows the competitiveness of the unstructured mesh model, given an efficient parallelization scheme.

\section{REMARKS}

The paper documents the development of an unstructured mesh, mesoscale model built on nonhydrostatic soundproof equations. The theoretical ideas are supported by numerical calculations of deep stratospheric gravity waves, breaking far from the excitation region. We have examined the solutions of pseudo-incompressible (Durran) and anelastic (LippsHemler) systems of governing equations, and shown that for a deep atmosphere with realistic density and potential temperature stratifications the results obtained from the two systems of equations exhibit differences at the level of higher order truncation errors. The solutions are equally insensitive to a choice of various shapes of unstructured meshes or types of traditional structured grid discretizations, provided that a similar spatial resolution is maintained. This supplies additional numerical evidence supporting earlier studies of weakly stratified mesoscale flows, by documenting the equivalence between the results obtained using the anelastic and pseudo-incompressible systems of equations. The application studied has been further modified, up to the point when the differences between the solutions could be clearly observed, although the physical conditions of such modified application have become unrealistic. The solution of the pseudo-incompressible system is thus identified as a potential practical measure for estimating the validity of anelastic equations for this class of atmospheric flows. The developments presented are more general than the specific application discussed. They represent ways of constructing nonoscillatory forward-in-time, MPDATA edge-based, unstructured mesh finite volume solvers for a wide range of governing PDE systems.

\section{Acknowledgments}

Comments from two anonymous referees helped to improve the presentation. This work was supported in part by the NERC award NE/G004358 and DOE award DE-FG0208ER64535. The National Center for Atmospheric Research is sponsored by the National Science Foundation. 


\section{References}

Achatz U., R. Klein, and F. Senf (2010), Gravity waves, scale asymptotics and the pseudoincompressible equations, J. Fluid Mech., 663, 120-147.

Axelsson 0. (1994), Iterative Solution Methods, Cambridge University Press, pp. 654.

Bacon D.P., N.N. Ahmad, Z. Boybeyi, T.J. Dunn, M.S. Hall, P.C.S. Lee, R.A. Sarma, M.D. Turner, K.T. Waight, S.H. Young, and J.W. Zack (2000), A Dynamically Adapting Weather and Dispersion model: The Operational Multiscale Environment Model with Grid Adaptivity (OMEGA), Month. Weather Rev., 128, 2044-2067.

Bacon D.P., N.N. Ahmad, T.J. Dunn, M.C. Monteith, and A. Sarma (2008): An operational multiscale system for hazards prediction, mapping, and response, Natural Hazards, 44, 317-327.

Barth T.J. (1992), Aspects of unstructured grids and finite volume solvers for the Euler and Navier-Stokes equations. In Special Course on Unstructured Grid Methods for Advection Dominated Flows AGARD Report 787; 6.1-6.61.

Behrens J., S. Reich, M. Laüter, B. Wingate, and D. Williamson (2010), The 2010 Workshop on the Solution of PDEs on the Sphere; http://www.awipotsdam.de/pde2010/pdes2010_program.pdf

Birkhoff G. and R.E. Lynch (1984), Numerical Solution of Elliptic Problems, SIAM, pp. 319.

Bunge H-P, M.A. Richards, and J.R. Baumgardner (1997), A sensitivity study of threedimensional spherical mantle convection at $10^{8}$ Rayleigh number: Effects of depthdependent viscosity, heating mode, and an endothermic phase change, J. Geophys. Res., 102, 11,991-12,007

Davies H.C. (1983), Limitations of some common lateral boundary schemes in regional NWP models, Month. Weather Rev., 111, 1002-1012.

Davies T., A. Staniforth, N. Wood, and J. Thuburn (2003), Validity of anelastic and other equation sets as inferred from normal-mode analysis, Q.J.R. Meteorol. Soc., 129, 27612775.

Doyle J.D., D.R. Durran, C. Chen, et al. (2000), An intercomparison of model-predicted wave breaking for the 11 January 1972 Boulder Windstorm, Month. Weather Rev., 128, 901-914.

Durran D.R. (1989), Improving the anelastic approximation. J. Atmos. Sci., 46, 1453-1461. 
Durran D.R. (2008), A physically motivated approach for filtering acoustic waves from the equations governing compressible stratified flow. J. Fluid Mech., 601, 365-379.

Eisenstat S.C., Elman H.C., and Schultz M.H. (1983), Variational iterative methods for nonsymmetric systems of linear equations, SIAM J. Numer. Anal., 20, 345-357.

Ghizaru M., Charbonneau P., and Smolarkiewicz P.K. (2010), Magnetic cycles in global large-eddy simulations of solar convection, Astrophys. J. Lett., 715, L133.

Grabowski W.W., and P.K. Smolarkiewicz (2002), A multiscale anelastic model for meteorological research, Month. Weather Rev., 130, 939-956.

Greenbaum A. (2002), Iterative Methods for Solving Linear Systems, SIAM, pp. 220.

Klein R., U. Achatz, D. Bresch, O.M. Knio, and P.K. Smolarkiewicz (2010), Regime of validity of soundproof atmospheric flow models, J. Atmos. Sci., 67, 3226-3237.

Klein R (2011), On the regime of validity of sound-proof model equations for atmospheric flows. Proceedings of the ECMWF Workshop on Nonhydrostatic Modelling, November 8-10, 2010, Reading, UK, in press.

Kosloff R., and D. Kosloff (1986), Absorbing boundaries for wave propagation problems, J. Comput. Phys., 63, 363-376.

Lipps F.B., and R.S. Hemler (1982), A scale analysis of deep moist convection and some related numerical calculations, J. Atmos. Sci., 39, 2192-2210.

Lipps F.B. (1990), On the anelastic approximation for deep convection, J. Atmos. Sci., 47, 1794-1798.

Miller M.J. and P.K. Smolarkiewicz (2008), Predicting weather, climate and extreme events, Preface, J. Comput. Phys., 227, 3429-3430.

Nikiforakis N. (2009), Mesh generation and mesh adaptation for large-scale Earth-system modelling, Introduction, Phil. Trans. R. Soc. A, 367, 4473-4481.

Piotrowski Z.P., A.A. Wysogrodzki, and P.K. Smolarkiewicz (2011), Towards petascale simulation of atmospheric circulations with soundproof equations, Acta Geophys., ibidem.

Prusa J.M., P.K. Smolarkiewicz, and R.R. Garcia (1996), Propagation and breaking at high altitudes of gravity waves excited by tropospheric forcing, J. Atmos. Sci., 53, 2186-2116.

Prusa J.M., P.K. Smolarkiewicz, and A.A Wyszogrodzki (2001), Simulations of gravity wave induced turbulence using 512 PE Cray T3E, Int. J. Appl. Math. Comp. Sci., 11, 883-897. 
Prusa J.M., and P.K. Smolarkiewicz (2003), An all-scale anelastic model for geophysical flows: dynamic grid deformation, J. Comput. Phys., 190, 601-622.

Prusa J.M., P.K. Smolarkiewicz, and A.A Wyszogrodzki (2008), EULAG, a computational model for multiscale flows, Comput. Fluids, 37, 1193-1207.

Saad Y. (1995), Iterative methods for sparse linear systems, PWS Publishing Company, pp. 447.

Smith R.B. (1979), The influence of the mountains on the atmosphere, Advances in Geophysics, 21, 87-230.

Smolarkiewicz P.K. (1983), A Simple Positive Definite Advection Scheme with Small Implicit Diffusion, Month. Weather Rev., 111, 479-486.

Smolarkiewicz P.K. (1984), A Fully Multidimensional Positive Definite Advection Transport Algorithm with Small Implicit Diffusion, J. Comput. Phys., 54, 325-362.

Smolarkiewicz P.K, and L.G. Margolin (1993), On forward-in-time differencing for fluids: extension to a curvilinear framework, Month. Weather Rev., 121, 1847-1859.

Smolarkiewicz, P.K., and L.G. Margolin (1994): Variational solver for elliptic problems in atmospheric flows, Appl. Math. \& Comp. Sci., 4, 527-551.

Smolarkiewicz P.K., V. Grubišić, and L.G. Margolin (1997), On forward-in-time differencing for fluids: stopping criteria for iterative solutions of anelastic pressure equations, Month. Weather Rev., 125, 647-654.

Smolarkiewicz P.K., and L.G. Margolin (1997), On forward-in-time differencing for fluids: An Eulerian/semi-Lagrangian nonhydrostatic model for stratified flows, Atmos. Ocean Special, 35, 127-152.

Smolarkiewicz PK, and L.G. Margolin (2000), Variational methods for elliptic problems in fluid models, Proc. ECMWF Workshop on Developments in numerical methods for very high resolution global models 5-7 June 2000; Reading, UK, ECMWF, 137-159.

Smolarkiewicz P.K., L.G. Margolin, and A.A Wyszogrodzki (2001), A class of nonhydrostatic global models, J. Atmos. Sci., 58, 349-364.

Smolarkiewicz P.K., C. Temperton, S.J. Thomas, and A.A. Wyszogrodzki (2004), Spectral Preconditioners for nonhydrostatic atmospheric models: extreme applications, Proceedings of the ECMWF Seminar Series on Recent developments in numerical methods for atmospheric and ocean modelling, Reading, UK, 203-220). 
Smolarkiewicz P.K., and J. Szmelter (2005a), Multidimensional positive definite advection transport algorithm (MPDATA): An edge-based unstructured-data formulation, Int. J. Numer. Meth. Fluids, 47, 1293-1299.

Smolarkiewicz P.K., and J. Szmelter (2005b), MPDATA: An edge-based unstructured-grid formulation, J. Comput. Phys., 206, 624-649.

Smolarkiewicz P.K. (2006), Multidimensional positive definite advection transport algorithm: an overview, Int. J. Numer. Meth. Fluids, 50, 1123-1144.

Smolarkiewicz P.K., R. Sharman, J. Weil, S.G. Perry, D. Heist, and G. Bowker (2007), Building resolving large-eddy simulations and comparison with wind tunnel experiments, J. Comput. Phys., 227, 633-653.

Smolarkiewicz P.K., and A. Dörnbrack, Conservative integrals of adiabatic Durran's equations, Int. J. Numer. Meth. Fluids, 56, 1513-1519.

Smolarkiewicz P.K., and J. Szmelter (2009), Iterated upwind schemes for gas dynamics, J. Comput. Phys., 228, 33-54.

Smolarkiewicz P.K., and C.L. Winter (2010), Pores resolving simulation of Darcy flows, J. Comput. Phys., 229, 3121-3133

Smolarkiewicz P.K. (2011), Modeling atmospheric circulations with soundproof equations. Proceedings of the ECMWF Workshop on Nonhydrostatic Modelling, November 8-10, 2010, Reading, UK, in press.

Szmelter J. and P.K. Smolarkiewicz (2006), MPDATA error estimator for mesh adaptivity, Int. J. Numer. Methods Fluids, 50, 1269-1293.

Szmelter J., and P.K. Smolarkiewicz (2010), An edge-based unstructured mesh discretisation in geospherical framework, J. Comput. Phys., 229, 4980-4995

Szmelter J., and P.K. Smolarkiewicz (2011), An edge-based unstructured mesh framework for atmospheric flows, Comput. Fluids, 46, 455-460.

Warn-Varnas A., J. Hawkins, P.K. Smolarkiewicz, S.A. Chin-Bing, D. King, and Z. Hallock (2007), Solitary wave effects north of Strait of Messina, Ocean Modelling, 18, 97-121.

Wedi N.P., and P.K. Smolarkiewicz (2004), Extending Gal-Chen \& Somerville terrainfollowing coordinate transformation on time-dependent curvilinear boundaries, J. Comput. Phys., 193, 1-20. 
Williamson D.L. (2008), The evolution of dynamical cores for global atmospheric models, J. Meteor. Soc. Japan, 85B, 241-268.

Wurtele M.G., R.D. Sharman and A. Datta (1996), Atmospheric lee waves, Annu. Rev. Fluid. Mech., 28, 429-476. 